\title{
Novartis reclaims tk technology
}

In what could be seen as part of a strategy to reclaim part of the technology that the US Federal Trade Commission (FTC, Washington, DC) forced it to outlicense, Novartis (Basel, Switzerland) has sought and been granted an exclusive license to gene therapy vectors that elude the immune system by Alexion Pharmaceuticals (New Haven, CT). Novartis will pay Alexion up to $\$ 10$ million in license, research, and milestone fees.

Alexion's retroviral vectors express an $\alpha$ 1,2-fucosyl transferase gene, the enzyme product of which produces a fucose-containing human carbohydrate epitope on the vector surface. Because the enzyme outcompetes $\alpha-1,3$-galactosyl transferase, the level of the $\alpha$-gal epitope that normally initiates the complement cascade leading to elimination of the vector is greatly reduced. According to Alexion president and CEO Leonard Bell, the number of $\alpha$-gal epitopes is reduced by over $99 \%$, and this allows the vectors to elude the immune response and persist in vivo for over a day.

Bell believes that Alexion's system could extend the scope of Novartis' thymidine kinase/ganciclovir (tk) cancer gene therapy, the approach that Novartis licensed to Rhône-Poulenc Rorer (Collegeville, PA) in December, at the FTC's insistence. Novartis is currently in phase III glioblastoma trials with the approach, the most advanced stage of clinical development of the technology. In the brain, the absence of immune cells and the lack of MHC molecules on brain cell surfaces permit persistence of the tk vectors, but elsewhere in the body, vectors can be eliminated in minutes, before they can integrate into growing cells. Bell says that such elimination is "the critical limiting factor" in extending the Novartis' technology to other cancers, such as those of the colon or ovaries, "and that's where our technology potentially has a role," he adds.

Scott Freeman, medical director of the blood center at Tulane University Medical School (New Orleans, LA), is already using tk vectors in treating metastatic ovarian cancer. $\mathrm{He}$ believes that Alexion's system is "an important technology [that] will be useful in maximizing the use of retroviral vectors," but he would not characterize it as a critical limiting step. In the phase I clinical trials he is running, which use infusions of tumor cells containing the tk gene but not modified to avoid immune elimination, 3 out of 16 patients are still in remission 2.5 years after the start of the trial. He believes that other issues, such as producing adequate distribution of the tk gene within solid tumors or increasing the efficiency of retroviral transduction, are as important.

One intriguing aspect of the deal with Alexion is its potential for strengthening Novartis' position in gene therapy. By acquiring exclusive rights to Alexion's "critical" technology-and, perhaps in the future, to similar approaches that achieve the same ends-Novartis could, in effect, reestablish a monopoly position in its own outlicensed technology.
Elizabeth Silverman, biotechnology analyst at Punk, Knoell and Ziegell (New York), characterizes the move as a continuation of Novartis' technology collecting. "It probably wasn't in their best interests that they had to share [the gene therapy technologies] but. . I think they would have continued building anyway." She sees the Alexion system as "potentially important technology" that Novartis will use to make its retrovirus vectors more powerful.

Emma Johnson

\section{Microbial genomics: Milestones mount exponentially}

Microbial genomic analysis is currently enjoying "exponential growth," according to J. Craig Venter, head of The Institute for Genomic Research (TIGR, Rockville, MD), at a conference* on small genomes held in late January. During the past two years, Venter calculates about a half dozen microbial genomes have been completed, and 1997 could see another 8-10 sequenced in their entirety.

The most recent addition is the Escherichia coli genome. Containing $4,638,858$ base pairs and 4,286 open reading frames, the sequence was completed by Fred Blattner at the University of Wisconsin (Madison, WI) and his colleagues over a fiveyear period. In hindsight, Blattner says that the project would have gone more quickly had his team followed the now-preferred strategy of "random-shotgun sequencing" of the genome. Indeed, by adopting this strategy, the Japanese E. coli Sequencing Group (JESG) was able to sequence most of the genome of another strain of this bacterium much more quickly, according to Hirotada Mori of the Nara Institute of Science and Technology (Nara, Japan). Reportedly, though, around $35 \%$ of the JESG sequence is drawn from Blattner's data (Nature 385: 472). Human Genome Sciences (HGS, Rockville, MD) has also sequenced $E$. coli, a pathogenic strain in this instance, according to analyst Reijer Lenstra of Smith Barney (New York).

At least another 6-12 microbial pathogens are under close scrutiny (see Table 1 ). Sequences are also expected soon on the microbial pathogens that cause typhus, stomach ulcers, Lyme disease, and syphilis. In the past six months, the US National Institute for Allergy and Infectious Diseases (NIAID, Bethesda, MD) of the US National Institutes of Health (NIH, Bethesda, MD) is sponsoring sequence analyses of pathogens responsible for a variety of diseases, including tuberculosis, several forms of sexually transmitted diseases, and cholera. Another even more ambitious project-sequencing the 30-million base-pair genome of Plasmodium falciparum, the parasite responsible for malaria-is also underway, but is at least several years from completion, says Stephen Hoffman of the Naval Medical Research Institute (Rockville, MD), which is participating in the effort. Several biotechnology companies, including HGS and Genome Therapeutics (GT, Waltham, $\mathrm{MA}$ ), also focus in part on microbial pathogens. According to GT's Douglas Smith, his company plans to set up genome sequence databases for several pathogens "to do comparative analysis as an aid to drug discovery." And last year, HGS sequenced the Staphylococcus aureus genome, subsequently signing an agreement with MedImmune (Gaithersburg, MD) to collaborate on developing antibacterial vaccines and immunotherapeutics for infections caused by this and 